\title{
RSII RAPID-SEQUENCE INDUCTION OF ANAESTHESIA AND INTUBATION OF THE TRACHEA
}

\section{Bogumila Woloszczuk-Gebicka}

Department of Intensive Care and Toxicology, Chair of Rescue Medicine, Institute of Midwifery and Rescue Medicine, Faculty of Medicine, University of Rzeszów

\begin{abstract}
Rapid sequence induction and intubation (RSII) is the preferred method of tracheal intubation in emergency situations for patients presenting with a full stomach. The aim of RSII is to intubate the trachea within 60 seconds, without having to use bag-valve-mask ventilation to avoid air insufflation into the stomach. After preoxygenation and while cricoid pressure is applied, an induction dose of intravenous anaesthetic agent is administered and rapidly followed by a fast-acting muscle relaxant, and after 60 seconds tracheal intubation is performed.
\end{abstract}

Preoxygention increases apnoea tolerance. This is particularly important for infants and young children, and in patients who are in critical condition, obese or pregnant. Cricoid pressure (the Sellick manouver) is recommended to prevent regurgitation of the gastric contents to the throat.

Propofol or thiopental are routinely used for induction. Ketamine or etomidate may be used if propofol or thiopental administration is contraindicated. Succinylcholine or rocuronium are used to facilitate tracheal intubation. Poor jaw relaxation, patient resistance to a laryngoscope, closed or closing vocal cords, vigorous limb movements or sustained coughing after tube insertion are not clinically acceptable.

Modified rapid sequence induction, used in patients at risk of rapid development of hypoxaemia, allows gentle positive pressure ventilation after administration of the induction agent and muscle relaxant, but before the tracheal intubation.

If the attempt of intubation fails, sugammadex, $16 \mathrm{mg} / \mathrm{kg}$ body weight is recommended for the immediate reversal of the neuromuscular block produced by rocuronium, but not for other muscle relaxants.

KEY WORDS: intubation endotracheal, intubation methods, muscle relaxants depolarizing, muscle relaxants non-depolarizing, cyclodextrines

Disaster Emerg Med J 2017; (2)1: 33-38

\section{INTRODUCTION}

Endotracheal intubation is the cornerstone of airway management. It provides maximal protection against aspiration of the gastric content to the lungs and allows for positive pressure ventilation with a higher airway pressures than during ventilation via a facemask or the supraglottic airway. Therefore, it is considered to be the definitive method of securing the airway.
Indications for tracheal intubation in emergency situation include:

— Inability to maintain the patency of the upper airway;

— Inability to protect the airway against aspiration;

- Respiratory failure;

- Traumatic brain injury, Glasgow Coma Scale < 9;

- The expected worsening of the patient's condition that will eventually lead to respiratory fail- 
ure, the inability to maintain airway patency or protection against aspiration;

- Other indications.

\section{DEFINITION}

Rapid sequence induction and intubation (RSII) is the preferred method of tracheal intubation in emergency situations. The aim of RSII is to intubate the trachea without having to use bag-valve-mask (BVM) ventilation. Positive pressure ventilation is avoided until the airway is secured with an endotracheal tube, unless attempts at intubation are unsuccessful, or desaturation occurs. Apnoea may be poorly tolerated by patients with compromised respiratory status i.e. patients in critical state, obese or pregnant, or by patients with a high baseline oxygen requirement, or young children.

\section{INDICATIONS AND CONTRAINDICATIONS}

Avoiding of air insufflation into the stomach is particularly important for patients who have not fasted ("full stomach") and who are therefore at a greater risk of vomiting and aspiration of the gastric content to the lungs.

It must be noted RSII is not indicated in unconscious and apnoeic patients when immediate positive pressure ventilation using a self-inflating bag and facemask (BVM) and endotracheal intubation without any induction drugs or muscle relaxants is indicated, as stated in the resuscitation guidelines [1].

RSII may be also a bad choice if difficult intubation is anticipated. In this case the "difficult airway protocol" [2], with some modifications for a full stomach, may be more appropriate.

\section{PERFORMING RSII}

The patient's airway should is assessed in order to predict the difficulty of intubation. An ECG, pulse-oxymeter and blood pressure cuff should be employed. The equipment for the intubation, including laryngoscopes, endotracheal tubes, guidewires and suction must be selected and checked. Intravenous access must be obtained and drugs for the induction prepared in syringes.

After adequate preoxygenation and while cricoid pressure is being applied, an induction dose of intravenous anaesthetic agent is administered and rapidly followed by a fast-acting muscle relaxant. The trachea is intubated without any attempts of positive pressure ventilation, all the time maintaining the cricoid pressure. The objective is to achieve optimal intubating conditions rapidly and to minimize the time between the loss of consciousness and securing the airway with a cuffed endotracheal tube.

Cricoid pressure (the Sellick manouver) is a key component of RSII. It involves the application of pressure at the cricoid ring to occlude the upper oesophagus, thereby preventing regurgitation of gastric content into the pharynx [3]. Air entry into the stomach is prevented as long as a patent airway is maintained [4] The effectiveness of cricoid pressure is due to occlusion of the hypopharynx [5], therefore the position of the oesophagus, which may have a tendency to move rightwards, seems to be irrelevant. The recommended force is 10 newtons is a conscious patient, increased to $30 \mathrm{~N}$ after loss of consciousness [6]. Although properly applied cricoid pressure is effective in reducing the risk of aspiration, excessive force can obstruct the subglottic airway and interfere with mask ventilation or successful tracheal intubation, worsening laryngeal visualization during a direct laryngoscopy, potentially extending the time to intubation and increasing the risk of pulmonary aspiration.

Using cricoid pressure is encouraged for RSII. If glottic visualization proves difficult, it can be easily released [7]. Using a videolaryngoscope seems to facilitate the selection of the optimal cricoid pressure.

Preoxygenation - a period of spontaneous breathing with $100 \%$ oxygen through a tightly fitted facemask in order to replace nitrogen in the lungs with oxygen before the administration of an induction agent and muscle relaxant, is recommended before the induction of general anaesthesia [7], as difficulty with airway management may occur unexpectedly. RRSI should also be preceded by preoxygenation whenever possible. Preoxygenation requires 3 minutes of normal breathing $[7,8]$ or a few very deep breaths (with vital capacity). Although the latter method may be more appropriate in an emergency, it requires the patient's cooperation. Preoxygention increases the apnoea tolerance, and gives more time to expose the larynx and place the tube in the trachea. It is particularly important for infants and young children who are less tolerant to apnoea due to increased oxygen consumption, and in patients who are in a critical condition, obese or pregnant. 


\section{DRUGS USED FOR RSII}

\section{Induction drugs}

RSII involves administration of an induction agent (i.e. propofol, thiopental, ketamine, etomidate) to render the patient unconscious, and a fast-acting muscle relaxant to achieve muscle relaxation and tracheal intubation within 60 seconds. It requires experienced, well trained medical staff (Tab. 1).

The dose of propofol or thiopental must be reduced in patients with hypovolemia, since with the "regular" adult dose, its concentration in the blood is higher than intended, hence the risk of circulatory collapse.

Ketamine is the drug of choice for patients in shock. Although its onset time is longer, which means that it takes longer from the injection to the loss of consciousness, blood pressure is maintained, as well as airway patency and spontaneous breathing.

Etomidate is the drug of choice for the patients with heart failure. Its onset time is long, with involuntary movements resembling seizures.

\section{Muscle relaxants}

Apart from the anatomy, intubating conditions depend on the masseter relaxation (jaw opening) and vocal cord relaxation. Scoring of the intubating conditions according to the Good Clinical Practice in Anaesthesia Guidelines [9] is presented in Table 2.

Intubating conditions are rated excellentif all qualities are excellent; good - if all qualities are excellent or good, and poorif any quality is poor. Excellent and good intubating conditions are summarised as clinically acceptable conditions. Poor jaw relaxation, patient resistance to a laryngoscope, closed or closing vocal cords, vigorous movements of the limbs or sustained coughing after the tube insertion are considered clinically not acceptable [9].

From its introduction the 1950s until recently, succinylcholine was the drug of choice in order to facilitate tracheal intubation. In recent years, however, it has largely been replaced by rocuronium.

Succinylcholine is still popular in emergency medicine as it has the fastest onset and shortest duration of action of all muscle relaxants, while excellent-to-good intubating conditions are usually achieved after $60 \mathrm{~s}$. Increasing age is associated with a slower onset of the neuromuscular block. After administration of $1.0 \mathrm{mg} / \mathrm{kg}$ body weight of succinylcholine, the onset time (the time from injection to the maximum effect) is $58 \pm 7 \mathrm{~s}$ in infants $1-3$ years old, and $95 \pm 7$ s in 60-80 year-old patients, respec-

\begin{tabular}{|c|c|c|c|c|}
\hline Agent & $\begin{array}{l}\text { "Typical adult" dose } \\
\text { (mg.kg-1) }\end{array}$ & Onset time to $\mathrm{LOC}^{*}$ & Main indications & Contraindications \\
\hline Propofol & 2 & Short & Standard RSII & Shock \\
\hline Thiopental & 5 & Short & Standard RSII & Shock \\
\hline Ketamine & $1-2$ & Long & Shock & Standard RSII \\
\hline Etomidate & $0.2-0.3$ & Long & Heart failure & Standard RSII \\
\hline
\end{tabular}

* LOC - loss of consciousness

\begin{tabular}{|l|c|c|c|}
\hline \multirow{2}{*}{ Table 2. Scoring conditions for tracheal intubation } & \multicolumn{2}{|c|}{ Intubating scores } \\
\cline { 2 - 4 } & \multicolumn{2}{|c|}{ Clinically acceptable } & \multicolumn{1}{|c|}{ Clinically not acceptable } \\
\hline Variable & Excellent & Good & Poor \\
\hline Laryngoscopy & & & Poor \\
\hline Jaw relaxation & Relaxed & Not fully & Active \\
\hline Resistance to laryngoscope & None & Slight & Closed \\
\hline Vocal cords & & & Closing \\
\hline Position & Abducted & Intermediate & \\
\hline Movement & None & Moving & Vigorous \\
\hline Reaction to tube insertion or cuff inflation & & & Sustained \\
\hline Movement of limbs & None & Slight & \\
\hline Coughing & None & Slight & \\
\hline
\end{tabular}


tively [10]. The time to regular spontaneous breathing is approx. 6 min. - which is too long, if the patient cannot be ventilated, while there are no drugs to speed up the return of spontaneous breathing. The time to or $90 \%$ recovery of the muscle strength is approx. 10 minutes [11].

The mechanism of action of succinylcholine is different from all other currently used muscle relaxants. Succinylcholine is called a "depolarizing" muscle relaxant, since its action resembles that of the natural mediator, acetylcholine. Succinylcholine combines with the acetylcholine receptor, opening the ion channels, which means depolarization of the neuromuscular junction and muscle fibres, and $\mathrm{Na}+$ and $\mathrm{K}+$ ion shift between the extracellular and intracellular fluid. Muscle relaxation is preceded by uncoordinated contractions of skeletal muscles - fasciculations, which appear first on the eyelids, soon afterwards on the neck (m. platysma), then in the chest muscles, abdomen, and the extremities. Fasciculations may produce muscle pain, possibly secondary to muscle damage produced by the unsynchronized contractions of muscle fibres just before the onset of paralysis. Although administration of a small dose of a non-depolarizing muscle relaxant ("precurarization") prevents fasciculations, and the muscle pain from succinylocholine is at least attenuated, it stops the swallowing reflex and may increase the risk of aspiration if regurgitation occurs in spite of cricoid pressure.

Succinylcholine administration may temporarily increase the masseter tone both in adults [12] and in children [13]. In patients with neuromuscular diseases, hemiplegia or paraplegia resulting from a cerebrovascular accident or trauma, muscular dystrophies, Guillain-Barré syndrome, extensive burns and severe injuries succinylcholine may cause significant hyperkaliemia, resulting in arrhythmia or cardiac arrest. Significant hyperkaliemia usually does not occur until several days after the injury. In the absence of infection or persistent degeneration of the tissue, patients are susceptible to hyperkaliemic response probably for at least 60 days after a massive trauma.

Succinylcholine usually causes an increase in intraocular pressure (IOP [14]). As long lasting (1-2 $\mathrm{min}$ ) contracture of the extraocular muscles pulls the eye against the orbit, succinylcholine should not be given to patients with open eye injuries [15]. It also has the potential to increase intracranial pressure (ICP [16]). In humans under light anaesthesia, ICP goes up by approx. $5 \mathrm{~mm} \mathrm{Hg}$ [17].
A rare, but life threatening complication of succinylcholine is malignant hyperthermia. This may occur in people with muscle dystrophies - a rare inherited muscle abnormality. As most cases occur in children, mainly boys, who are unaware of the condition, it is advised to limit using succinylcholine in children. With prompt treatment using dantrolene, malignant hyperthermia usually resolves itself within 12-24 h [18].

In the recent years, succinylcholine has been largely replaced by rocuronium, which is a non-depolarizing, or competitive muscle relaxant. Rocuronium combines with the acetylcholine receptor at the neuromuscular junction, but does not activate it, which means that there is no membrane depolarization, no ion shift, no fasciculations, no muscle damage, and no risk related to hyperkaliemia or malignant hyperthermia. For RSII, 1.0 mg rocuronium bromide per $\mathrm{kg}$ body weight is recommended for facilitating tracheal intubation [19]. Adequate intubation conditions are established within 60 seconds in nearly all (> 90\%) patients, and rated "excellent" in $70 \%$ of patients [19]. Increasing the intubation dose to $1.2 \mathrm{mg} / \mathrm{kg}$ body weight increases the probability of successful intubation at $60 \mathrm{~s}$ [20]. It should be noted that the rocuronium dose recommended for the RSII is higher than the dose of 0.6 used during routine anaesthesia. Conditions associated with prolonged circulation time such as cardiovascular diseases, old age and an generalized oedema resulting in an increased volume of distribution may contribute to a slower onset of the effect. When used in overweight or obese patients (defined as patients with a body weight of $30 \%$ or more above ideal body weight) doses should be reduced, taking into account a lean body mass [21].

\section{FAILED INTUBATION AND REVERSAL OF THE NEUROMUSCULAR BLOCK PRODUCED BY ROCURONIUM}

The clinical duration of the neuromuscular block after $1.0 \mathrm{mg} / \mathrm{kg}$ body weight of rocuronium is much longer than that of succinylcholine. The time from the administration of $1.06 \mathrm{mg} / \mathrm{kg}$ body weight of rocuronium to the first detectable symptoms of the recovery of neuromuscular transmission is $46 \mathrm{~min}$ utes. A few minutes later, muscle strength can be restored with an acetylcholinesterase inhibitor, neostigmine. If neostigmine is administered after the onset of spontaneous recovery of the neuromus- 
cular transmission, the recovery of muscle strength takes 9-13 minutes [22]. However, if neostigmine is administered too early, before the onset of the spontaneous recovery, it will be inefficient. Therefore it cannot be used for the rapid restoration of spontaneous breathing after a failed attempt at intubation. Sugammadex (Bridion, N.V. Organon, The Netherlands) is a modern reversal agent offering the possibility of rapidly reversing the profound neuromuscular block produced by rocuronium. Sugammadex is the first selective relaxant binding agent. It has no effect on acetylcholinesterases and its mechanism of action is completely different from neostigmine. Sugammadex molecules encapsulate rocuronium molecules in plasma and in the extracellular fluid forming an inactive complex. This creates a concentration gradient between the rocuronium concentration in the neuromuscular junction and plasma and rapid diffusion of the muscle relaxant from the acetylcholine receptors to the extracellular fluid and plasma. This restores neuromuscular transmission and muscle strength. The inactive complex of rocuronium and sugammadex is excreted by the kidneys [23].

For the immediate reversal of a rocuroniuminduced neuromuscular block, a dose of $16 \mathrm{mg} / \mathrm{kg}$ body weight of sugammadex is recommended - a dose many times higher than that routinely used at the end of anaesthesia. It is administered as a single bolus injection, given rapidly into an existing venous line. When $16 \mathrm{mg} / \mathrm{kg}$ body weight of sugammadex is given 3 minutes after a bolus dose of $1.2 \mathrm{mg}$ of rocuronium, the median recovery time to the moment when the patient regains muscle strength is approximately 1.5 minutes. In obese patients, the dose of sugammadex should be based on actual body weight. Immediate reversal in children and adolescents has not been investigated and is therefore not recommended by the manufacturer until further data becomes available. The decision to use sugammadex "off-label" in a paediatric patient is left at the discretion of the clinician, who must weigh the potential benefits and risks [24].

Sugammadex is not recommended for immediate reversal of the neuromuscular block produced by succinylcholine or any other muscle relaxants, vecuronium included.

On rare occasions, marked bradycardia, sometimes resulting in cardiac arrest, has been observed within minutes after sugammadex administration. Monitoring of haemodynamic changes, and atro- pine administration, if clinically indicated, are therefore recommended.

It must be noted that muscle relaxants do not produce unconsciousness or pain relief. As muscle paralysis without anaesthesia causes considerable psychological distress which the patient is unable to communicate, administration of the muscle relaxants to a conscious patient is contraindicated.

\section{Latency}

During RSII, the injection site important. If the induction drugs and muscle relaxant are administered to a small peripheral vein, especially in the lower extremities, the time required to reach the brain and neuromuscular junction is much longer than after the administration to larger veins, which is equivalent to a long latency. If small peripheral veins must be used, the drug should be flushed with an appropriate volume of a fluid, usually $0.9 \%$ saline.

Latency will also be long in patients with slow circulation, i.e. those with heart failure or hypothermia.

\section{MODIFIED RAPID SEQUENCE INDUCTION}

Modified rapid sequence induction consists of preoxygenation, the application of cricoid pressure, and gentle positive pressure ventilation after administration of the induction agent and muscle relaxant, but before the tracheal intubation. Indications for this technique include patients at risk of rapid development of hypoxaemia (e.g., in those critically ill, morbidly obese or pregnant), in emergency situations where preoxygenation cannot be satisfactorily completed or when a longer time to achieve tracheal intubation is anticipated. Although the effect of positive pressure ventilation with the application of cricoid pressure in terms of gastric insufflation of air is not definitively known, gentle positive pressure ventilation with an inspiratory pressure $<20 \mathrm{~cm} \mathrm{H}_{2} \mathrm{O}$ in combination with cricoid pressure may be acceptable in these clinical scenarios [25]. Positive pressure ventilation via a face mask may also be provided before and after administration of a muscle relaxant for patients unable to tolerate the brief period of apnoea associated with RSII.

To summarize, rapid sequence induction and intubation is the method of choice for emergency intubation. It requires careful planning and skilful execution. In the case of failed intubation, sugammadex may be used to restore muscle strength and spontaneous breathing. 
Funding sources: None declared. Conflict of interest: None declared.

\section{REFERENCES}

1. https://cprguidelines.eu/.

2. https://www.das.uk.com/guidelines/das_intubation_guidelines.

3. Sellick BA. Cricoid pressure to control regurgitation of stomach contents during induction of anaesthesia. Lancet. 1961; 2(7199): 404-406, indexed in Pubmed: 13749923.

4. Lawes EG, Campbell I, Mercer D. Inflation pressure, gastric insufflation and rapid sequence induction. Br J Anaesth. 1987; 59(3): 315-318, indexed in Pubmed: 3828180.

5. Rice MJ, Mancuso AA, Gibbs C, et al. Cricoid pressure results in compression of the postcricoid hypopharynx: the esophageal position is irrelevant. Anesth Analg. 2009; 109(5): 1546-1552, doi: 10.1213/ ane.0b013e3181b05404, indexed in Pubmed: 19843793.

6. Vanner RG, Asai T. Safe use of cricoid pressure. Anaesthesia. 1999; 54(1): 1-3, indexed in Pubmed: 10209362.

7. Hagberg CA, Artime CA. Airway management in the adult. In: Miller RD. ed. Miller's Anesthesia. Eight Edition. Elsevier, Philadelphia 2014: 1647-1687.

8. Thwaites AJ, Rice CP, Smith I. Rapid sequence induction: a questionnaire survey of its routine conduct and continued management during a failed intubation. Anaesthesia. 1999; 54(4): 376-381, indexed in Pubmed: 10455840.

9. Fuchs-Buder T, Claudius C, Skovgaard LT, et al. 8th International Neuromuscular Meeting. Good clinical research practice in pharmacodynamic studies of neuromuscular blocking agents II: the Stockholm revision. Acta Anaesthesiol Scand. 2007; 51(7): 789-808, doi: 10.1111/j.13996576.2007.01352.x, indexed in Pubmed: 17635389.

10. Koscielniak-Nielsen ZJ, Bevan JC, Popovic V, et al. Onset of maximum neuromuscular block following succinylcholine or vecuronium in four age groups. Anesthesiology. 1993; 79(2): 229-234, indexed in Pubmed: 8102040.

11. El-Orbany MI, Joseph NJ, Salem MR, et al. The neuromuscular effects and tracheal intubation conditions after small doses of succinylcholine. Anesth Analg. 2004; 98(6): 1680-1685, indexed in Pubmed: 15155328.

12. Leary NP, Ellis FR. Masseteric muscle spasm as a normal response to suxamethonium. Br J Anaesth. 1990; 64(4): 488-492, indexed in Pubmed: 2334624.
13. Meakin G, Walker RW, Dearlove OR. Myotonic and neuromuscular blocking effects of increased doses of suxamethonium in infants and children. Br J Anaesth. 1990; 65(6): 816-818, indexed in Pubmed: 2265051.

14. Pandey K, Badola RP, Kumar S. Time course of intraocular hypertension produced by suxamethonium. Br J Anaesth. 1972; 44(2): 191-196, indexed in Pubmed: 5016887.

15. Martyn JA, Richtsfeld M. Succinylcholine-induced hyperkalemia in acquired pathologic states: etiologic factors and molecular mechanisms. Anesthesiology. 2006; 104(1): 158-169, indexed in Pubmed: 16394702.

16. Minton MD, Grosslight $K$, Stirt JA, et al. Increases in intracranial pressure from succinylcholine: prevention by prior nondepolarizing blockade. Anesthesiology. 1986; 65(2): 165-169, indexed in Pubmed: 2874752.

17. Patel PM, Drummond JC, Lemkuil BP. Cerebral physiology and the effects of anesthetic drugs. In: Miller RD. ed. Miller's Anesthesia. Eight Edition. Elsevier, Philadelphia 2014: 387-422.

18. https://www.drugs.com/health-guide/malignant-hyperthermia.html.

19. http://www.medicines.org.uk/emc/medicine/23095/SPC.

20. Kirkegaard-Nielsen H, Caldwell JE, Berry PD. Rapid Tracheal Intubation with Rocuronium. A probability approach to determining dose. Anesthesiology. 1999; 91(1): 131-136, doi: 10.1097/00000542199907000-00021.

21. http://www.medicines.org.uk/emc/medicine/23095/SPC.

22. Jones RK, Caldwell JE, Brull SJ, et al. Reversal of profound rocuronium-induced blockade with sugammadex: a randomized comparison with neostigmine. Anesthesiology. 2008; 109(5): 816-824, doi: 10.1097/ALN.0b013e31818a3fee, indexed in Pubmed: 18946293.

23. Schaller SJ, Fink H. Sugammadex as a reversal agent for neuromuscular block: an evidence-based review. Core Evid. 2013; 8: 57-67, doi: 10.2147/CE.S35675, indexed in Pubmed: 24098155.

24. Wołoszczuk-Gębicka B, Zawadzka-Głos L, Lenarczyk J, et al. Two cases of the "cannot ventilate, cannot intubate" scenario in children in view of recent recommendations. Anaesthesiol Intensive Ther. 2014; 46(2): 88-91, doi: 10.5603/AlT.2014.0017, indexed in Pubmed: 24858967.

25. Clements $P$, Washington SJ, McCluskey $A$. Should patients be manually ventilated during rapid sequence induction of anaesthesia? $\mathrm{Br} J$ Hosp Med (Lond). 2009; 70(7): 424, doi: 10.12968/hmed.2009.70.7.43138, indexed in Pubmed: 19584792. 